\title{
Introduction to the special section on interventional abdominal radiology
}

\author{
Fred T. Lee Jr., Timothy J. Ziemlewicz $\odot$ \\ Department of Radiology, University of Wisconsin, 600 Highland Ave, MC 3252, Madison, WI 53792, USA
}

\begin{abstract}
Abdominal radiologists have an illustrious history in the invention and development of image-guided percutaneous interventional techniques. Many of the most common procedures performed in radiology departments worldwide were first described by abdominal radiologists, including percutaneous biopsy, abscess drainage, and percutaneous tumor ablation. Unfortunately, for various reasons, some abdominal radiologists have been for-
\end{abstract} ced - or have chosen - to forgo performing these procedures, often for reasons of convenience or turf. This change in focus may even be subconsciously reflected in a change in title, from "abdominal radiologist" to "abdominal imager" or even the antiquated misnomer: "body imager." We believe that this is unfortunate in that true excellence in image-guided procedures arises from a deep understanding of the anatomy, disease processes, state-ofthe-art imaging, and clinical skills; all attributes of abdominal radiologists. We want to be clear-procedural expertise is not the exclusive domain of any particular subspecialty, but by virtue of the daily viewing of thousands of images with all imaging technologies, responsibility for the technical aspects of image acquisition, interaction with multiple referring subspecialists and patients, and extensive knowledge of the origins and consequences of abdominal diseases, abdominal radiologists have all the tools necessary to provide excellence in imageguided abdominal interventions.

The Society of Abdominal Radiology has acted recently to reinforce the axiom that the study and dissemination of knowledge about percutaneous interventions in the abdomen will remain a critical part of the society moving forward. To reflect this renewed emphasis, the official society journal has been renamed the Journal of Abdominal Radiology (JAR) to reflect a more comprehensive approach to the abdomen as the founders initially envisioned. Indeed, the inaugural issue of this journal, published as "Gastrointestinal Radiology" in 1976, contained a report by Melson et al. entitled "Selective intraarterial infusion of

Correspondence to: Fred T. Lee Jr.; email: flee@uwhealth.org vasopressin for control of gastrointestinal bleeding: Experience with 35 cases" [1]. The editor-in-chief of the JAR, Dr. C. Daniel Johnson, has also appointed an associate editor for image-guided interventions, Dr. Fred Lee $\mathrm{Jr}$., as well as several editorial board members well known for their expertise and innovation in interventional techniques. We would urge authors who are studying percutaneous image-guided interventions in the abdomen, regardless of their self-identified specialty or country of origin, to consider JAR as a forum for their work.

We are pleased to present this special edition of JAR focused on image-guided interventions in the abdomen. Leading abdominal radiologists from throughout the United States, Asia, and Europe have submitted articles covering the gamut of percutaneous interventions performed worldwide. Besides outstanding scientific and review articles, this special edition includes an opinion piece on a future vision for abdominal intervention from Dr. Silverman and colleagues, and an editorial on the role of percutaneous biopsy in the work-up of renal masses authored by a premier urologic oncologist, Dr. E. Jason Abel.

We are thankful to the authors who contributed their expertise to this special edition and are gratified by the high level of work which is occurring in our field. The future of intervention in abdominal radiology is strong and will continue to grow due to the dedication of the many talented physicians in this field. We hope that the information provided in this issue will serve as a wellreferenced resource on the state-of the-art in intervention for years to come.

Compliance with Ethical Standards

Conflict of interest Fred T. Lee, Jr, MD: Shareholder, patent holder and board of directors, Neuwave Medical Inc, Madison WI; Patent holder, inventor, royalties, Covidien AG. Timothy J. Ziemlewicz: No disclosures.

\section{Reference}

1. Melson GL, Geisse G, Stanley RJ (1976) Selective intraarterial infusion of vasopressin for control of gastrointestinal bleeding: experience with 35 cases. Gastrointest Radiol 1(1):59-65 